\title{
An investigation into the factors which affect the sitting balance of non-ambulatory children with cerebral palsy
}

\author{
Damla Cankurtaran $m D$, Nihan Abidin $M D$, Ece Unlu Akyuz $M D$, Nihal Tezel $M D$ \\ Department of Physical Medicine and Rehabilitation, Diskapi Yildirim Beyazit Education and Research \\ Hospital, Ankara, Turkey
}

\begin{abstract}
Background \& Objective: This study aimed to examine the factors affecting sitting function in nonambulatory children with cerebral palsy (CP) who spend most of their daily life in a sitting position. Methods: Thirty- five non-ambulatory children with $\mathrm{CP}$ were included in this cross sectional study. Data included demographics, socio-economic features, examination findings, personal and family history, and accompanying problems of patients. The participants' lower extremity spasticity was evaluated using the Modified Ashworth scale (MAS); manual ability was evaluated using the Manual Ability Classification System (MACS); sitting function using the Gross Motor Functional Measure (GMFM)-sitting subscale; and trunk control using the Trunk Impairment Scale (TIS). The relationship between sitting function and the other evaluation parameters was analyzed. Results: Children with scoliosis had significantly lower scores in TIS-static and TIS-total $(\mathrm{p}<0.05)$. Hip problems negatively affected the GMFM-sitting subscale, TIS-static, and TIS-total $(\mathrm{p}<0.05)$. Strong positive correlations were found between GMFM- B (sitting subscale) scores and TIS-static, dynamic, coordination, and TIS-total scores $(\mathrm{p}<0.05)$. Duration of hospitalization in neonatal intensive care and MACS were correlated with GMFM-B ( $\mathrm{p}<0.05)$. Multivariate analyses showed that trunk control and upper extremity function were independent risk factors on sitting function.

Conclusion: More attention should be paid to rehabilitation programs which incorporate trunk control exercises and upper extremity functions in order to improve sitting function. Preventing hip dislocation may be an option to increase sitting function of non-ambulatory children with CP.
\end{abstract}

Keywords: cerebral palsy, non-ambulatory, sitting function, spasticity, trunk control

\section{INTRODUCTION}

Cerebral palsy (CP) describes a group of permanent disorders related to the development of movement and posture which caused activity limitation and are attributed to non-progressive disturbances that occur in the developing fetal or infant brain. ${ }^{1}$ Motor disorders can be accompanied by sensory and cognitive disorders, communication, perception, epilepsy, behavioral disorders, and secondary musculoskeletal problems. ${ }^{1}$ Interactions between the central and peripheral nervous systems and the musculoskeletal system, which is necessary for stable posture, are affected in children with $\mathrm{CP} .{ }^{2}$ Hyperactive stretch reflexes, poor selective motor control, and dysregulation between muscle groups due to brain damage cause impaired trunk control. ${ }^{2}$
Optimal trunk control is necessary for daily activities such as sitting or reaching in children with CP. ${ }^{3}$ Sitting postural control is the earliest upright posture achieved in childhood development. Sitting independently provides the possibility of active arm use, more potential for functional skills and self-care, and opportunities to orient the self to the environment for improved perception, cognitive growth, and social interaction. ${ }^{4}$ Sitting balance is also important for predicting the ability to walk in children with $\mathrm{CP} .^{5}$

Rehabilitation programs focus on walking ability in children with CP. However, children with moderate to severe motor impairments, such as those at levels IV and V of the Gross Motor Function Classification System (GMFCS), are typically unable to stand independently and walk. ${ }^{6}$ They spend most of their time in sitting

Address correspondence to: Damla Cankurtaran, MD, Department of Physical Medicine and Rehabilitation, Diskapi Yildirim Beyazit Education and Research Hospital, 06080 Altındag- Ankara, Turkey. Tel; +90-5054558101. E-mail: damlacengizftr@gmail.com

Date of Submission: 8 March 2021; Date of Acceptance: 21 May 2021

https://doi.org/10.54029/2021xua 
positions and their trunk control is weak. ${ }^{2}$ Postural control is an important part of the therapeutic goals in rehabilitation programs for children with $\mathrm{CP}$, especially for children who cannot walk independently. ${ }^{7}$ As such, it may be helpful for clinicians who work with $\mathrm{CP}$ patients to understand which factors affect trunk control and sitting function when planning rehabilitation programs.

Previous studies have demonstrated that trunk control is an important factor on functionality, especially sitting function in CP. ${ }^{2}$ However, the factors associated with sitting function and trunk control have been investigated in children with ambulatory and non-ambulatory $\mathrm{CP}$ together. This study aimed to examine the factors affecting sitting functions in non-ambulatory children with $\mathrm{CP}$ who spend most of their daily life in sitting positions.

\section{METHODS}

This study was designed as a cross-sectional study. Study protocol approval was obtained from the local ethics committee at our hospital (Decision no: 90/07), and the parents of participant children gave written informed consent to join the research and allow publication of the results. The study was performed according to principles of the Declaration of Helsinki.

\section{Participants}

Thirty-five children who met the inclusion criteria participated in this study. The inclusion criteria for this study were: 1) diagnosis of spastic CP, 2) aged between 5-18 years, 3 ) unable to walk independently or with assistance, 4) able to sit with support or sit without support for short periods (30 seconds), and 5) able to follow simple instructions. Children who could walk independently or with assistance, or who had a lower limb fracture or muscle-tendon/bone surgery in the last six months, severe mental retardation at a level hindering communication, or a baclofen pump, or who had been exposed to any focal pharmacological spasticity treatment (Phenol or Botulinum toxin-A) in the last three months were excluded from study.

\section{Patients'data}

Socio-demographic features age (years), gender, gestational age at birth, birth weight ( $\mathrm{g}$ ), duration of treatment in neonatal intensive care unit (days), diagnosis time (month), age of mother at birth (years), mother and father's education level and job, primary caregiver, prenatal, natal, or postnatal risk factors, family history, and accompanying problems which were previously diagnosed by a physician (e.g., speech disorders, visual disorders, swallowing disorders, hearing disorders, mental retardation, drooling, dental problems, scoliosis, hip problems, urinary incontinence, stool incontinence, and epilepsy) were noted.

\section{Spasticity evaluation}

The spasticity of the lower extremity muscles (gastrocnemius (GC), hamstring and hip adductors) were evaluated bilaterally using the Modified Ashworth Scale (MAS) ${ }^{8}$ The MAS is a commonly used scale for measuring muscle tone during passive movement with a 6-point scale: 0 $=$ no increased resistance, $1=$ lightly increased resistance (minimal resistance at the end of the range of motion or catch follow by relaxation) $1+$ $=$ slightly increased resistance (catch follow by resistance throughout less than half of range of motion ), 2 = clear resistance throughout most of the range of motion, $3=$ strong resistance, passive motion is difficult, $4=$ rigid flexion or extension. ${ }^{9}$

During the examination, the children were lying in a supine position with the head in a neutral position without a pillow and the lower extremities extended and parallel to the trunk. The measurements were made without clothing.

\section{Manual ability}

Manual ability was examined using the Manual Ability Classification System (MACS), which consists of 5 levels of classification according to how well children with $\mathrm{CP}$ use their hands to handle objects in daily activities. Level 1 describes best manual activity in daily life, and level 5 represents children who cannot perform basic hand functions. ${ }^{10}$

To evaluate the sitting function and trunk control of the patients, the gross motor function measure (GMFM)-88 (Section B, Sitting) and Trunk Impairment Scale (TIS) (static, dynamic, coordination, total score) were used.

\section{Gross motor function measure-88}

The GMFM- 88 is the most commonly used scale to evaluate motor development in children with $\mathrm{CP}$, and it is considered to be a valid, reliable, and useful tool. ${ }^{11}$ It contains five sections and 88 items: (1) lying and rolling (17 items), (2) sitting (20 items), (3) crawling and kneeling (14 items), (4) standing (13 items), and (5) walking, 
running, and jumping (24 items). A four-point Likert scale is used to score according to the child's accomplishment level. The total score of the GMFM- 88 is obtained by adding up the total points for each section. A total score or the scores of each section can be used separately. ${ }^{12}$ In our study, we used Section B (Sitting) to measure sitting function.

\section{Trunk Impairment Scale}

The trunk control of patients was evaluated using the TIS, which was first developed and used for stroke patients. ${ }^{13}$ Since then, the validity and reliability of this scale for patients with $\mathrm{CP}$ has been demonstrated. ${ }^{14}$ This scale consists of three sub-sections: static balance, dynamic balance, and coordination. Task scores for each activity range from 0 to 2 . The total score ranges from 0 to 23 . Higher total scores indicate better trunk control in sitting posture. ${ }^{14}$

\section{Study Protocol}

Patients' data were collected before physical evaluation. One physician evaluated the patients while another physician, who was blind to the patients' data, evaluated the participants using the MAS, MACS, GMFM-88 sitting subscale, and TIS. The relationship between sitting function, trunk control, socio-demographic features, personal history, family history, spasticity, and manual ability was examined.

\section{Statistical analysis}

Statistical Package for the Social Sciences (SPSS 20.0 for Windows) software package was used for data analysis. The variables were investigated using visual (histograms, probability plots) and analytical methods (Shapiro- Wilk's test) to determine whether or not they were normally distributed. For descriptive statistics, the data were expressed as mean \pm standard deviation (SD) for continuous and categorical variables, and as frequencies and percentages (\%) for nominal variables. Non-normally distributed numerical variables were compared using Mann-Whitney U tests for two groups and Kruskal Wallis test for more than two groups. Correlations were analyzed using Spearman's or Pearson correlation tests where required. Simple and multiple linear regression analysis (backward modeling) were carried out to determine the most important predictor(s) for explaining GMFM-B variance. A model using MACS, TIS-total, duration of hospitalization in neonatal intensive care unit, presence of scoliosis, and hip problems was analyzed. Statistical significance was considered for $\mathrm{p}$ values less than 0.05 .

\section{RESULTS}

Thirty-five patients, consisting of 15 (42.9\%) girls and $20(57.1 \%)$ boys, with CP were included in the study. The median age of the patients was 8 years old (range 5-15 years). The median gestational age at birth was 32 weeks (range 26-40 weeks) a median birth weight of $1815.16 \pm 779.12 \mathrm{~g}$. The mean duration of treatment in neonatal intensive care units was $34.35 \pm 35.11$ weeks, and the median diagnosis time was 8 months (range 3-24 months), and the mothers' mean age at birth was $27.71 \pm 5.62$ years

No significant correlation was found between the results of the GMFM-B and mothers' education level, fathers' education level, mother's job, and fathers' job ( $p>0.05)$. Participants with different prenatal, natal, postnatal risk factors and family history had statistically similar results from the GMFM- B ( $>>0.05)$.

Table 1 shows the comparison of sitting function and trunk control between patients with accompanying problems. Children with scoliosis had significantly lower scores on the TIS-static and TIS-total ( $\mathrm{p}=0.02, \mathrm{p}=0.04$, respectively). Hip problems negatively affected patients' GMFMsitting subscale, TIS-static, and TIS-total scores ( $\mathrm{p}=0.02, \mathrm{p}=0.04, \mathrm{p}=0.04$, respectively).

Significant correlations between the GMFM-B (sitting subscale) and evaluation parameters are shown in Table 2.

Positive correlations were found between GMFM-B (sitting subscale) scores and TIS-static, -dynamic, -coordination, and TIS-total scores $(\mathrm{r}=0.789, \mathrm{p}=0.001 ; \mathrm{r}=0.745, \mathrm{p}=0.001 ; \mathrm{r}=0.618$, $\mathrm{p}=0.001 ; \mathrm{r}=0.863, \mathrm{p}=0.001$, respectively).

Duration of hospitalization in neonatal intensive care and MACS were significantly correlated with GMFM-B (sitting subscale; $r=-$ $0.392, p=0.024 ; \mathrm{r}=-0.403, \mathrm{p}=0.016$, respectively). Age, gender, gestational age at birth, birth weight, diagnosis time, and age of mother at birth were not correlated with GMFM-B ( $\mathrm{p}>0.05)$.

According to the correlation analysis, no significant correlation was found between GMFM-B and MAS-gastrocnemius right/left, MAS-hamstring right/left, and MAS-hip adductor right/left ( $\mathrm{p}>0.05)$.

Table 3 shows the results of univariate and multivariate regression analyses. MACS and TIS- 


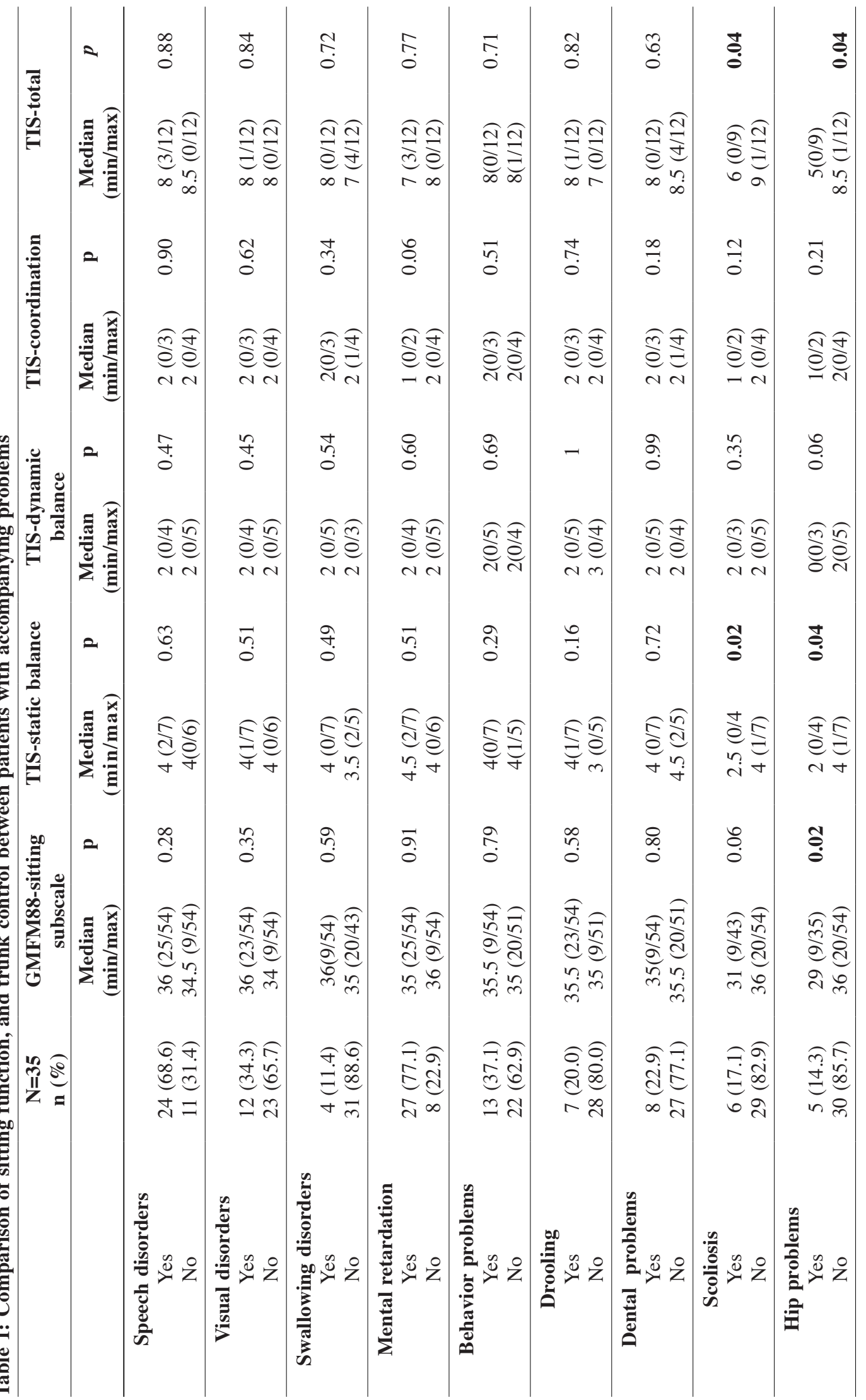


total were found to be independent risk factors of sitting function according to the multivariate analyses $(\beta=-0.20,(-6.49 /-0.21) \mathrm{p}=0.04 ; \beta=0.78$, $(1.77 / 3.01), \mathrm{p}<0.001$ respectively).

\section{DISCUSSION}

The factors affecting sitting function in only children with non-ambulatory $\mathrm{CP}$ were examined using various measurement tools. Trunk control and upper extremity functions were determined to be a significant predictor of sitting function in non-ambulatory children with $\mathrm{CP}$ with $79.1 \%$ variance in this study. The findings of this study revealed that patients with hip dislocation had worse sitting functions and trunk control than those without hip dislocation, and patients with scoliosis had worse trunk control than those without with scoliosis. Duration of hospitalization in a neonatal intensive care unit was correlated with sitting function. No association was found between spasticity of the lower extremity muscles.

The relationship between trunk control and functional activities like sitting in children with $\mathrm{CP}$ is an issue that researchers have studied. ${ }^{2,14}$ In a recent study, Kallem at al. investigated the relationship between trunk control and functionality in 25 children with $\mathrm{CP}$; their study included both ambulatory and non-ambulatory children. They found that the total score of GMFM-88 was significantly correlated with the total score of TIS $(r=0.78, p<0.01)$. Moreover, the sitting subscale of GMFM-88 was positively correlated with TIS-static sitting balance, TISdynamic balance, and TIS-coordination ( $\mathrm{r}=0.809$, $\mathrm{r}=0.429, \mathrm{r}=0.432$, respectively). ${ }^{2}$ In another study, total TIS scores and all TIS sub-scores were found to be significantly correlated with the GMFM88 sitting subscale with correlation coefficients varying between 0.62 and $0.87 . .^{14}$ Curtis et al. reported that the segmental level of trunk control is one of the important factors determining gross motor function and mobility skills for children with $\mathrm{CP}$, predicting between $30 \%$ and $40 \%$ of the variance in GMFM. ${ }^{15}$ However, they did not analyze the effect of segmental level of trunk control involved in sitting function only; instead, all gross motor functions were evaluated together. ${ }^{15}$

The participants in our study consisted of patients who were unable to walk, and we only analyzed the sitting subscale of GMFM. The other four subscales of the GMFM-88 were not evaluated, because we wanted to focus only on sitting function, which is more important 
Table 2: Results of Spearman correlation analyses

\begin{tabular}{lcc}
\hline & \multicolumn{1}{c}{$\mathbf{n = 3 5}$} \\
\hline GMFM-B & $\mathbf{R}$ & $\mathbf{P}$ \\
TIS-static balance & & 0.001 \\
TIS-dynamic & 0.789 & 0.001 \\
TIS-coordination & 0.745 & 0.001 \\
TIS-total & 0.618 & 0.001 \\
MACS & 0.863 & 0.016 \\
Duration of treatment in neonatal intensive care & -0.403 & 0.024 \\
\hline
\end{tabular}

GMFM-B: gross motor function measure sitting subscale, TIS: Trunk impairment scale. MACS: Manual Ability Classification System.

Table 3: Results of regression analyses

\begin{tabular}{|c|c|c|c|c|c|c|}
\hline & & \multicolumn{5}{|c|}{ Multivariate (Backward modeling) } \\
\hline & & 及 & $\mathbf{p}$ & $B$ & $\begin{array}{c}\text { Standard } \\
\text { error } B\end{array}$ & $\mathbf{R}^{2}$ \\
\hline \multirow[t]{6}{*}{1} & Constant & & $<0.001$ & 27.06 & 5.07 & \multirow{6}{*}{0.781} \\
\hline & MACS & $-0.20\left(-6.49 \_-0.21\right)$ & 0.038 & -3.35 & 1.54 & \\
\hline & TIS-total & 0.78(1.76_3.01) & 0.001 & 2.38 & 0.31 & \\
\hline & Hip problem & $-0.18\left(-14.76 \_3.61\right)$ & 0.226 & -5.48 & 4.42 & \\
\hline & Scoliosis & $0.09\left(-4.73 \_9.86\right)$ & 0.721 & 2.56 & 3.55 & \\
\hline & $\begin{array}{l}\text { Duration of } \\
\text { treatment in } \\
\text { neonatal } \\
\text { intensive care }\end{array}$ & $-0.01\left(-0.05 \_0.04\right)$ & 0.89 & -0.01 & 0.002 & \\
\hline \multirow[t]{5}{*}{2} & Constant & & $<0.001$ & 26.79 & 4.62 & \multirow{5}{*}{0.789} \\
\hline & MACS & $-0.19(-6.44-0.26)$ & 0.034 & -3.35 & 1.51 & \\
\hline & TIS-total & 0.78 (1.84_2.95) & 0.001 & 2.39 & 0.27 & \\
\hline & Hip problem & $-0.18\left(-14.31 \_3.44\right)$ & 0.220 & -5.43 & 4.33 & \\
\hline & Scoliosis & $0.09\left(-4.54 \_9.73\right)$ & 0.463 & 2.59 & 3.48 & \\
\hline \multirow[t]{4}{*}{3} & Constant & & $<0.001$ & 27.08 & 4.57 & \multirow{4}{*}{0.792} \\
\hline & MACS & $-0.19(-6.41-0.29)$ & 0.033 & -3.35 & 1.49 & \\
\hline & TIS-total & $0.78\left(1.83 \_2.93\right)$ & 0.001 & 2.38 & 0.26 & \\
\hline & Hip problem & $-0.09\left(-9.08 \_2.92\right)$ & 0.303 & -3.7 & 2.93 & \\
\hline \multirow[t]{3}{*}{4} & Constant & & $<0.001$ & 27.41 & 4.57 & \\
\hline & MACS & $-0.23(-6.77-1.06)$ & 0.009 & -3.91 & 1.39 & \multirow{2}{*}{0.791} \\
\hline & TIS-total & 0.81 (1.96_2.99) & 0.001 & 2.48 & 0.25 & \\
\hline
\end{tabular}

GMFM-B: gross motor function measure sitting subscale, TIS: Trunk impairment scale. MACS: Manual Ability Classification System, B: unstandardized regression coefficient; Std. Error B: standardized error of Beta; $\beta$ : standardized regression coefficient; R2: coefficient of determination. 
for non-ambulatory children with $\mathrm{CP}$ or other neuromuscular disease. In line with previous studies, we investigated trunk control as an independent factor on the sitting function of non-ambulatory children with CP. We determined that with an increase in the TIS-total score by one unit, there would be an increase of 0.81 in the GMFM-88 sitting subscale.

In our study, we found that upper extremity functions were a factor which affected sitting balance. We have determined that as the MACS increases by one level, the GMFM-88 sitting subscale scores will decrease by 0.23 . Children with better upper extremity functions can perform corrective protective and balance reactions more accurately and have better sitting function. ${ }^{16}$

In our study, we found that sitting function and trunk control were significantly worse in patients with hip dislocation than those without, and trunk control was significantly worse in patients with scoliosis than those without. Krebs et al. found that $14 \%$ of patients with hip dislocation had an improved sitting skill after surgery. ${ }^{17}$ A study found that patients with scoliosis had lower scores from the GMFM-88 total and sitting subscale than those without. ${ }^{18} \mathrm{Hip}$ dislocation and scoliosis are common in children with $\mathrm{CP}$, but it is not clear which deformity develops firsts. ${ }^{19}$ Progressive lateral dislocation of the femur from the acetabulum causes muscle imbalance due to spasticity in the hip flexors and adductors as well as weakness in the hip abductor and extensors. Another reason for hip dislocation is that the femoral anteversion angle remains high in children with CP. ${ }^{17,20}$ In children with non-ambulatory $\mathrm{CP}$, spasticity in the extremity muscles and weakness in the trunk muscles due to insufficient in postural control, and the subsequent inability of inhibition in vertebral abnormal growth patterns, cause disruptions in spine alignment. Spinal deformities increase with the continuation of this vicious cycle. ${ }^{21}$ One of the potential reasons why trunk control is worse in patients with scoliosis is weakness in the postural muscles. Children with hip dislocation and scoliosis have obliquity in the pelvis..$^{21}$ Asymmetric load transfer during sitting due to the disruption of the pelvis with horizontal correction may cause sitting function to worsen. That this study found patients with hip dislocation to be worse in terms of sitting function than those without hip dislocation may be attributed to the asymmetric load transfer that develops as a result of the disruption of the relationship of the pelvis with the horizontal plane after the above-mentioned mechanisms.
Spasticity adversely affects the functionality of children with non-ambulatory CP. The findings of previous studies concerning significant improvement in the GMFM- 88 total scores of children with non-ambulatory $\mathrm{CP}$ with botulinum toxin injections support this view. ${ }^{22}$ In our study, the spasticity of the hip adductor, knee flexor, and ankle plantar flexor muscles was evaluated separately for each muscle by MAS, and no relationship was found between the spasticity of each muscle and sitting balance. To our knowledge, no previous studies have examined the relationship between spasticity and only sitting function, especially in children with non-ambulatory $\mathrm{CP}$. In one study, a correlation coefficient of 0.64 was found between the GMFM-88 score and the mean score obtained by summing the hip flexor, knee flexor and extensor, and ankle plantar flexor spasticity MAS scores and dividing them by four. ${ }^{23}$ Balzer et al. found a weak correlation between lower extremity spasticity, total MAS score (0-48), and trunk control with a low correlation coefficient $(\mathrm{r}<-0.3)$ in ambulatory children with CP. ${ }^{24}$ They calculated the total MAS score by adding the MAS score of the bilateral hip flexor, hip extensor, knee flexor, knee extensor, and ankle plantar flexor. ${ }^{24}$ Another study found a relationship between the total MAS score of hip flexor, knee flexor and ankle plantar flexor spasticity and GMFM-66 scores..$^{25}$ In our study, we did not examine the relationship of mean MAS values or the total MAS value. Therefore, we may not be able to detect a relationship between spasticity and sitting balance in our study. In order to examine the effect of spasticity on sitting function, we think that it would be more appropriate to evaluate spasticity globally rather than for muscles separately. Future research can be conducted with more participants, and speed-dependent scales can be used.

In a study which analyzed the outcomes of 728 infants, the infants who had been on mechanic ventilators for more than 15 days had a higher risk of $\mathrm{CP}$ than those who had been on mechanic ventilators for less than two days. ${ }^{26}$ Infants with very low weight, very low gestational age, and severe neurologic damage were hospitalized longer in neonatal intensive care units, and they had worse functional outcomes. Although no correlation was found between sitting function and birth weight or gestational age, this study found a negative correlation between duration of hospitalization in neonatal intensive care units and sitting function.

The low number of participants in this study and the absence of a control group are two 
limitations. The fact that only non-ambulatory, short-term sitting, and children who could respond to commands were included children were included in the study contributed to the low number of participants.

In conclusion, non-ambulatory children with CP spend most of their day sitting, and good trunk control and sitting balance are required to prepare these children for ambulation. Trunk control and upper extremity function were predictive factors on sitting function in non-ambulatory children with CP. In order to increase sitting function, more attention should be paid to rehabilitation programs that incorporate trunk control and upper extremity exercises in non-ambulatory children with CP. Hip problems and scoliosis in non-ambulatory children with CP should be kept in mind as another factor that may affect sitting function and trunk control, and preventive rehabilitation programs should be given more importance.

\section{ACKNOWLEDGEMENTS}

We would like to thank our clinical staff and the patients who participated in this study.

\section{DISCLOSURE}

Conflict of interest: None

\section{REFERENCES}

1. Rosenbaum P, Paneth N, Leviton A, et al. A report: the definition and classification of cerebral palsy. Dev Med Child Neurol 2007 Suppl;109:8-14.

2. Kallem Seyyar G, Aras B, Aras O. Trunk control and functionality in children with spastic cerebral palsy. Dev Neurorehabil 2019;22:120-25.

3. Barbado D, Reina R, Roldan A, McCulloch K, et al. How much trunk control is affected in adults with moderate-to-severe cerebral palsy? J Biomech 2019; 82:368-74.

4. Harbourne RT, Willett S, Kyvelidou A, et al. A comparison of interventions for children with cerebral palsy to improve sitting postural control: a clinical trial. Phys Ther 2010;90(12):1881-98.

5. Bañas BB, Gorgon EJ. Clinimetric properties of sitting balance measures for children with cerebral palsy: a systematic review. Phys Occup Ther Pediatr 2014; 34:313-34.

6. Palisano RJ, Rosenbaum P, Bartlett D, et al. Content validity of the expanded and revised Gross Motor Function Classification System. Dev Med Child Neurol 2018; 50(10):744-50.

7. Pavão SL, Maeda DA, Corsi C, et al. Discriminant ability and criterion validity of the Trunk Impairment Scale for cerebral palsy. Disabil Rehabil 2019;41(18):2199-205.

8. Alhusaini AA, Dean CM, Crosbie J, et al. Evaluation of spasticity in children with cerebral palsy using
Ashworth and Tardieu Scales compared with laboratory measures. J Child Neurol 2010;25(10):1242-47.

9. Li F, Wu Y, Li X. Test-retest reliability and interrater reliability of the Modified Tardieu Scale and the Modified Ashworth Scale in hemiplegic patients with stroke. Eur J Phys Rehabil Med 2014;50:9-15.

10. Carnahan KD, Arner M, Hägglund G. Association between gross motor function (GMFCS) and manual ability (MACS) in children with cerebral palsy. A population-based study of 359 children. BMC Musculoskelet Disord 2014;21;8:50.

11. Palisano RJ, Hanna SE, Rosenbaum PL, et al. Validation of a model of gross motor function for children with cerebral palsy. Phys Ther 2000;80(10):974-85.

12. Beckers LW, Bastiaenen CH.Application of the Gross Motor Function Measure-66(GMFM-66) in Dutch clinical practice: a survey study. BMC Pediatrics 2015;15:146.

13. Verheyden G, Nieuwboer A, Mertin J, et al. The Trunk Impairment Scale: a new tool to measure motor impairment of the trunk after stroke. Clin Rehabil 2004;18(3):326-34.

14. Saether R, Helbostad JL, Adde L, et al. Reliability and validity of the Trunk Impairmen Scale in children and adolescents with cerebral palsy. Res Dev Disabil 2013;34(7):2075-84

15. Curtis DJ, Butler P, Saavedra S, et al. The central role of trunk control in the gross motor function of children with cerebral palsy: a retrospective cross-sectional study. Dev Med Child Neurol 2015;57(4):351-7.

16. Seyhan K, Kerem-Günel M. Does stable sitting influence upper limb function in children with cerebral palsy? Turk J Pediatr 2019;61:79-84.

17. Krebs A, Strobl WM, Grill F. Neurogenic hip dislocation in cerebral palsy: quality of life and results after hip reconstruction. $J$ Child Orthop 2018;2(2):125-31.

18. Bertoncelli CM, Solla F, Loughenbury PR, et al. Risk Factors for Developing Scoliosis in Cerebral Palsy: A Cross-Sectional Descriptive Study. J Child Neurol 2017;32(7):657-62.

19. Senaran H, Shah SA, Glutting JJ, et al. The associated effects of untreated unilateral hip dislocation in cerebral palsy scoliosis. J Pediatr Orthop 2006;26(6):769-72.

20. Kim IS, Park D, Ko JY, Ryu JS. Are seating systems with a medial knee support really helpful for hip displacement in children with spastic cerebral palsy GMFCS IV and V? Arch Phys Med Rehabil 2019;100(2):247-53.

21. Hasler C, Brunner R, Grundshtein A, et al. Spine deformities in patients with cerebral palsy; the role of the pelvis. J Child Orthop 2020;14(1):9-16.

22. Choi JY, Kim SK, Park ES. The Effect of Botulinum Toxin Injections on Gross Motor Function for Lower Limb Spasticity in Children with Cerebral Palsy. Toxins (Basel) 2019;11(11):651. doi: 10.3390/ toxins 11110651 .

23. Ostensjø S, Carlberg EB, Vøllestad NK. Motor impairments in young children with cerebral palsy: relationship to gross motor function and everyday activities. Dev Med Child Neurol 2004;46:580-89. 
24. Balzer J, Marsico P, Mitteregger E, et al. Influence of trunk control and lower extremity impairments on gait capacity in children with cerebral palsy. Disabil Rehabil 2018;40(26):3164-70.

25. Noble JJ, Gough M, Shortland AP. Selective motor control and gross motor function in bilateral spastic cerebral palsy. Dev Med Child Neurol 2019;61(1):5761.

26. Tsai WH, Hwang YS, Hung TY, et al. Association between mechanical ventilation and neurodevelopmental disorders in a nationwide cohort of extremely low birth weight infants. Res Dev Disabil 2014;35:1544-50. 